\title{
Universal correction for the Becke-Johnson exchange potential
}

\author{
E. Räsänen, 1,* S. Pittalis,,$^{2,3,4}$ and C. R. Proetto ${ }^{3,4, \dagger}$ \\ ${ }^{1}$ Nanoscience Center, Department of Physics, \\ University of Jyväskylä, FI-40014 Jyväskylä, Finland \\ ${ }^{2}$ Department of Physics and Astronomy, \\ University of Missouri, Columbia, Missouri 65211, USA \\ ${ }^{3}$ Institut für Theoretische Physik, Freie Universität Berlin, \\ Arnimallee 14, D-14195 Berlin, Germany \\ ${ }^{4}$ European Theoretical Spectroscopy Facility (ETSF)
}

(Dated: November 17, 2018)

\begin{abstract}
The Becke-Johnson exchange potential [J. Chem. Phys. 124, 221101 (2006)] has been successfully used in electronic structure calculations within density-functional theory. However, in its original form the potential may dramatically fail in systems with non-Coulombic external potentials, or in the presence of external magnetic or electric fields. Here, we provide a system-independent correction to the Becke-Johnson approximation by (i) enforcing its gauge-invariance and (ii) making it exact for any single-electron system. The resulting approximation is then better designed to deal with current-carrying states, and recovers the correct asymptotic behavior for systems with any number of electrons. Tests of the resulting corrected exchange potential show very good results for a Hydrogen chain in an electric field and for a four-electron harmonium in a magnetic field.
\end{abstract}

\footnotetext{
*Electronic address: erasanen@jyu.fi

†Permanent address: Centro Atómico Bariloche and Instituto Balseiro, 8400 S.C. de Bariloche, Río Negro, Argentina
} 
Electronic structure calculations are routinely carried out by using density-functional theory [1, 2] (DFT) and its variants. The accuracy of a DFT calculation depends on the approximation used for the exchange-correlation energy functional. Substantial efforts have been made in deriving accurate approximations over the past few decades [3].

Within spin-DFT (SDFT) the optimized-effective-potential (OEP) method [4 6] provides a rigorous access to the exact exchange (EXX) energy,

$$
E_{x}\left[\rho_{\sigma}\right]=-\frac{1}{2} \sum_{\sigma=\uparrow, \downarrow} \sum_{j, k=1}^{N_{\sigma}} \int d^{3} r \int d^{3} r^{\prime} \frac{\varphi_{j \sigma}^{*}(\mathbf{r}) \varphi_{k \sigma}^{*}\left(\mathbf{r}^{\prime}\right) \varphi_{j \sigma}\left(\mathbf{r}^{\prime}\right) \varphi_{k \sigma}(\mathbf{r})}{\left|\mathbf{r}-\mathbf{r}^{\prime}\right|},
$$

and to the Kohn-Sham (KS) exchange potential $v_{x \sigma}(\mathbf{r})=\delta E_{x} / \rho_{\sigma}(\mathbf{r})$. Hartree atomic units (a.u.) are used throughout. Above $\varphi_{j \sigma}(\mathbf{r})$ are the spin-dependent Kohn-Sham (KS) orbitals, with energies $\varepsilon_{j \sigma}$, and

$$
\rho_{\sigma}(\mathbf{r})=\sum_{j=1}^{N_{\sigma}}\left|\varphi_{j \sigma}(\mathbf{r})\right|^{2}
$$

is the ground-state density. The OEP method leads to an integral equation which can be solved iteratively together with the standard KS equations. The main origin of practical complexity in the OEP method are the orbital shifts containing unoccupied KS orbitals [7]. Despite great progress in solving the equations for various systems [8 -16], and in algorithmic developments [17, 18], efficient and accurate approximations reducing the numerical burden of the full OEP scheme are still needed.

A simple approximation for the exchange potential has been proposed by Becke and Johnson (BJ) [19]:

$$
\begin{aligned}
v_{x \sigma}^{\mathrm{BJ}}(\mathbf{r}) & =v_{x \sigma}^{\mathrm{SL}}(\mathbf{r})+\Delta v_{x \sigma}^{\mathrm{BJ}}(\mathbf{r}) \\
& =v_{x \sigma}^{\mathrm{SL}}(\mathbf{r})+C_{\Delta v}\left[\frac{\tau_{\sigma}(\mathbf{r})}{\rho_{\sigma}(\mathbf{r})}\right]^{1 / 2},
\end{aligned}
$$

where

$$
v_{x \sigma}^{\mathrm{SL}}(\mathbf{r})=-\frac{1}{\rho_{\sigma}(\mathbf{r})} \sum_{j, k=1}^{N_{\sigma}} \int d^{3} r^{\prime} \frac{\varphi_{j \sigma}^{*}(\mathbf{r}) \varphi_{k \sigma}^{*}\left(\mathbf{r}^{\prime}\right) \varphi_{j \sigma}\left(\mathbf{r}^{\prime}\right) \varphi_{k \sigma}(\mathbf{r})}{\left|\mathbf{r}-\mathbf{r}^{\prime}\right|},
$$

is the Slater potential,

$$
\tau_{\sigma}(\mathbf{r})=\sum_{j=1}^{N_{\sigma}}\left|\nabla \varphi_{j \sigma}(\mathbf{r})\right|^{2}
$$

is (twice) the spin-dependent kinetic-energy density, and $C_{\Delta v}=\left[5 /\left(12 \pi^{2}\right)\right]^{1 / 2}$. Note that the Slater potential in Eq. (4), which is now the only numerical bottleneck, could be alternatively approximated by the semi-local Becke-Roussel approach [20]. Interestingly, the exact 
exchange potential could be written as in Eq. (33), but with $\Delta v_{x \sigma}^{\mathrm{BJ}}(\mathbf{r})$ replaced by $\Delta v_{x \sigma}^{\mathrm{OEP}}(\mathbf{r})$, which can be decomposed into the so-called Krieger-Li-Iafrate (KLI) approximation [21], plus another correction given in terms of the orbital shifts [7]. As it is well known, in many cases the KLI approximation is in good agreement with the full OEP.

Despite the semi-locality of $\Delta v_{x \sigma}^{\mathrm{BJ}}(\mathbf{r})$ in Eq. (3), the BJ potential is able to correctly yield the step structure in the exchange potential of several atoms [19]. Moreover, it has recently been shown that the BJ potential correctly reproduces the derivative discontinuity for fractional particle numbers [22]. During the first few years after its introduction, the BJ approximation has already been applied to various systems [22 28]. Impressively, the band gap of a large variety of extended systems is extremely well reproduced [26, 28]. However, as we will demonstrate below, the BJ potential may dramatically fail in the presence of an electric or magnetic field, or a non-Coulombic external potential.

The limitation of the BJ potential originates from two facts: (i) it is not gauge-invariant and (ii) it is not exact for all possible one-electron systems. These two problems may be fixed in similar fashion as demonstrated in our recent derivation of a BJ-type approximation for two-dimensional systems [29].

Before proceeding further, we would like to comment on the gauge-invariance requirement. For systems acted upon an external vector potential, the exchange potential we propose in this work should be identified as an approximation derived for the exact exchange-potential obtained within current-spin-density functional theory [30, 31] (CSDFT) by taking the functional derivative of the exchange-correlation energy functional (written in terms of the spinparticle and vorticity density) at constant vorticity. On the other hand, it is clear that since $E_{x}$ in SDFT depends only on $\rho_{\sigma}(\mathbf{r})$, it must be a gauge-invariant quantity by definition. As a direct consequence, the corresponding $v_{x \sigma}(\mathbf{r})$ is gauge-invariant as well.

Therefore, we propose the following correction

$$
\begin{aligned}
v_{x \sigma}(\mathbf{r}) & =v_{x \sigma}^{\mathrm{SL}}(\mathbf{r})+\Delta v_{x \sigma}^{\mathrm{C}}(\mathbf{r}) \\
& =v_{x \sigma}^{\mathrm{SL}}(\mathbf{r})+C_{\Delta v}\left[\frac{D_{\sigma}(\mathbf{r})}{\rho_{\sigma}(\mathbf{r})}\right]^{1 / 2},
\end{aligned}
$$

with

$$
D_{\sigma}(\mathbf{r})=\tau_{\sigma}(\mathbf{r})-\frac{1}{4} \frac{\left(\nabla \rho_{\sigma}(\mathbf{r})\right)^{2}}{\rho_{\sigma}(\mathbf{r})}-\frac{\mathbf{j}_{p \sigma}^{2}(\mathbf{r})}{\rho_{\sigma}(\mathbf{r})}
$$


where

$$
\mathbf{j}_{p \sigma}(\mathbf{r})=\frac{1}{2 i} \sum_{j=1}^{N_{\sigma}}\left[\varphi_{j \sigma}^{*}(\mathbf{r})\left(\nabla \varphi_{j \sigma}(\mathbf{r})\right)-\left(\nabla \varphi_{j \sigma}^{*}(\mathbf{r})\right) \varphi_{j \sigma}(\mathbf{r})\right]
$$

is the spin-dependent paramagnetic current density. The above potential in Eq. (6) has a set of desirable properties listed below.

- In contrast with $\tau_{\sigma}(\mathbf{r})$, as it appears in the BJ expression, the combination $\tau_{\sigma}(\mathbf{r})-$ $\mathbf{j}_{p \sigma}^{2}(\mathbf{r}) / \rho_{\sigma}(\mathbf{r})$ is clearly gauge-invariant. [32 34] As a result, also the corresponding potential is gauge-invariant.

- In contrast with $\Delta v_{x \sigma}^{\mathrm{BJ}}, \Delta v_{x \sigma}^{\mathrm{C}}$ is zero for all one-particle systems. This is easy to see by considering an arbitrary one-particle system with $\rho_{\sigma}(\mathbf{r})=\left|\varphi_{\sigma}(\mathbf{r})\right|^{2}$ and $\varphi_{\sigma}(\mathbf{r})=$ $\sqrt{\rho_{\sigma}(\mathbf{r})} e^{i \theta(\mathbf{r})}$, so that $D_{\sigma}(\mathbf{r}) \equiv 0$ follows immediately from Eq. (77). Alternatively, this may been seen by using the definition of $\tau_{\sigma}(\mathbf{r})$ and $\mathbf{j}_{p \sigma}(\mathbf{r})$ [Eqs. (5) and (8) , respectively] in terms of $\varphi_{\sigma}(\mathbf{r})$, and by re-expressing $\rho_{\sigma}(\mathbf{r})=\left|\varphi_{\sigma}(\mathbf{r})\right|^{2}$ in the second term of $\Delta v_{x \sigma}^{\mathrm{C}}$.

- The asymptotic limit is correct for any $N$-electron finite system: $\Delta v_{x \sigma}^{\mathrm{C}}(\mathbf{r} \rightarrow \infty) \rightarrow 0$ and then $v_{x \sigma}(\mathbf{r} \rightarrow \infty) \rightarrow v_{x \sigma}^{\mathrm{SL}}(\mathbf{r} \rightarrow \infty) \rightarrow-1 / r$. In that limit all the terms in $D_{\sigma}$ are dominated by the highest occupied orbital [35], and thus the system effectively behaves like a one-particle system (see the preceding point). Below we discuss the asymptotic limit in detail for two particular systems.

- Equation (6) is consistent with the limit of the homogeneous 3D electron gas (3DEG): $\Delta v_{x \sigma}^{\mathrm{OEP}}=\Delta v_{x \sigma}^{\mathrm{BJ}}=\Delta v_{x \sigma}^{\mathrm{C}}=\left[3 \rho_{\sigma} /(4 \pi)\right]^{1 / 3}$.

- Calculation of $\Delta v_{x \sigma}^{\mathrm{C}}$ instead of $\Delta v_{x \sigma}^{\mathrm{BJ}}$ does not bring any extra computational burden.

- Also, it is reassuring to note that, the exchange potential in Eq. (6) scales linearly as the exact one (see Appendix) [36].

Finally we point out that the key object in the corrected exchange potential, $D_{\sigma}(\mathbf{r})$, is familiar from various concepts in the literature. First, it is an important part of the electron localization function [37-39], and second, it enters in the expression of the local curvature of the exchange hole [40]. In the latter case, it is a part of the current-generalized forms [41-43] of the Becke-Roussel and Becke models for the exchange [20] and correlation [44], respectively. 


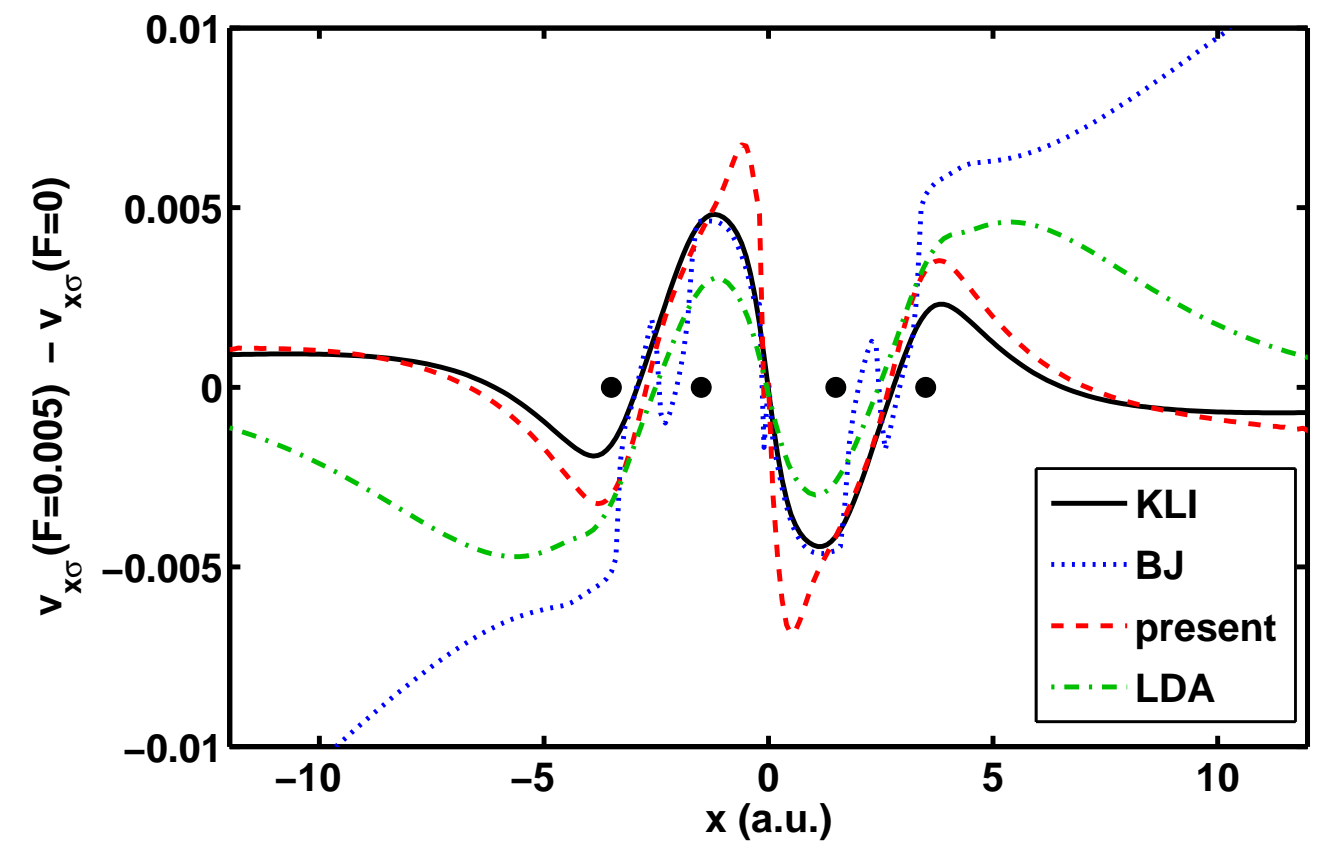

FIG. 1: (Color online) Difference in the exchange potentials for a four atom hydrogen chain with and without the linear electric field. Black dots denote the positions of Hydrogen atoms.

Next we test our exchange potential against the KLI [21], BJ, and local-density approximation (LDA) for two different systems. We perform the self-consistent KLI calculations applying the octopus [45] DFT code on a real-space grid. The resulting KS orbitals are then used as inputs in the approximations for the exchange potentials.

First we consider a $H_{4}$ chain in an external linear field with the same system parameters as in the work by Armiento, Kümmel, and Körzdörfer [22] (AKK). The system consists of two $\mathrm{H}_{2}$ "molecules" with an interpair distance of 2 a.u. separated by 3 a.u. The strength of the electric field, applied along the $x$ direction, is $F=0.005$ a.u. (hartree/bohr). Figure 1 shows the difference in the exchange potential with and without the electric field, i.e., $v_{x \sigma}(F=$ $0.005)-v_{x \sigma}(F=0)$. Overall, we find excellent agreement between KLI (solid line) and the present approximation (dashed line) in Eq. (6).

Figure 11 can be directly compared to Figs. 3 and 5 in Ref. [22]. We find the same divergence in the BJ potential in the asymptotic regime, as well as the large deviation of the LDA from the KLI result. More importantly, we find that our corrected formula is closer to 
KLI as the potential proposed by AKK, i.e.,

$$
\begin{aligned}
& v_{x \sigma}^{\mathrm{AKK}}(\mathbf{r})=v_{x \sigma}^{\mathrm{SL}}(\mathbf{r})+ \\
& C_{\Delta v}\left(\sqrt{\frac{\tau_{\sigma}(\mathbf{r})}{\rho_{\sigma}(\mathbf{r})}}-\sqrt{-2 \varepsilon_{N_{\sigma} \sigma}}-\frac{x F}{\sqrt{-2 \varepsilon_{N_{\sigma} \sigma}}}\right),
\end{aligned}
$$

with $\varepsilon_{N_{\sigma} \sigma}$ corresponding to the energy of the highest occupied KS orbital [46]. In particular, the AKK potential difference is close to zero on the left $(x \lesssim-10)$, whereas the KLI and the present approximation yield a finite value in that regime. Close to the atoms we find some overestimation in the maxima (and minima) of our potential, but, on the other hand, our approximation is free from sharp kinks present in the BJ and AKK potential differences.

Next we compare in detail the asymptotic limit of the above example given by the AKK potential [Eq. (99)] and our potential [Eq. (66)], respectively. For atomic systems in the absence of external fields the asymptotic behavior of the KS orbitals is given by [7]

$$
\varphi_{j \sigma}(\mathbf{r}) \stackrel{\mathbf{r} \rightarrow \infty}{\longrightarrow} \Phi_{j \sigma}(r) f_{j \sigma}(\Omega)
$$

with $\Phi_{j \sigma}(r)$ being the asymptotic radial wavefunction, and $f_{j \sigma}(\Omega)$ its corresponding angular component. The asymptotic form of $\Phi_{j \sigma}(r)$ is [7]

$$
\Phi_{j \sigma}(r) \stackrel{r \rightarrow \infty}{\longrightarrow} r^{1 / \beta_{j \sigma}} \frac{e^{-\beta_{j \sigma} r}}{r},
$$

with $\beta_{j \sigma}=\sqrt{-2 \varepsilon_{j \sigma}}$. Substituting Eqs. (10) and (11) into Eq. (6), it is easy to find that the leading correction in the asymptotic limit is given by

$$
\begin{aligned}
v_{x \sigma}(\mathbf{r}) \stackrel{\mathbf{r} \rightarrow \infty}{\longrightarrow} & v_{x \sigma}^{\mathrm{SL}}(\mathbf{r}) \\
& +C_{\Delta v}\left[\frac{\tau_{\sigma}(\mathbf{r})}{\rho_{\sigma}(\mathbf{r})}-\left(-2 \varepsilon_{N_{\sigma} \sigma}\right)+g_{N_{\sigma} \sigma}(\Omega)\right]^{1 / 2},
\end{aligned}
$$

where $g_{N_{\sigma} \sigma}(\Omega)=-\left[\nabla f_{N_{\sigma} \sigma}(\Omega)\right]\left[\nabla f_{N_{\sigma} \sigma}^{*}(\Omega)\right] /\left|f_{N_{\sigma} \sigma}(\Omega)\right|^{2}$ is a purely angular term with contributions coming from the second and third terms in $D_{\sigma}$ defined in Eq. (7). Making the subsequent asymptotic expansion of $\tau_{\sigma}(\mathbf{r}) / \rho_{\sigma}(\mathbf{r}) \stackrel{\mathbf{r} \rightarrow \infty}{\longrightarrow}-2 \epsilon_{N_{\sigma} \sigma}-g_{N_{\sigma} \sigma}(\Omega)$, it is apparent that the term inside the square-root in Eq. (12) vanishes identically in the asymptotic regime. We emphasize that, Eq. (12) is similar, but not identical, to the AKK potential in Eq. (9), with $F=0$. The main difference is that while the satisfaction of several exact constraints (as explained above) enforces us to have all the contributions of $D_{\sigma}$ inside the square-root, the correction in the AKK potential that enforces the vanishing of the exchange potential in the asymptotic limit $\left(\sqrt{-2 \epsilon_{N_{\sigma} \sigma}}\right)$ is outside the square-root. 
Similar considerations apply when the external electric field $F$ is present. Following AKK, in this case the asymptotic behavior of the $\mathrm{KS}$ atomic orbitals along the direction of the applied electric field is given by [47]

$$
\varphi_{j \sigma}(x) \stackrel{x \rightarrow \infty}{\longrightarrow} \eta^{-1 / 4} e^{-2 \eta^{3 / 2} / 3}
$$

with $\eta=(2 F)^{1 / 3}\left(x-\varepsilon_{j \sigma} / F\right)$. Proceeding with the evaluation of $\Delta v_{x \sigma}^{\mathrm{C}}(x \rightarrow \infty)$, we find

$$
\Delta v_{x \sigma}^{\mathrm{C}}(x) \stackrel{x \rightarrow \infty}{\longrightarrow} C_{\Delta v}\left[\frac{\tau_{\sigma}(x)}{\rho_{\sigma}(x)}-2\left(x F-\varepsilon_{N_{\sigma} \sigma}\right)\right]^{1 / 2}
$$

which again is similar, but not identical, to Eq. (9). Making them identical would require an expansion of the argument inside the square-root, but this is unjustified, since in the asymptotic regime both contributions are equally important. That is, $\tau_{\sigma}(x) / \rho_{\sigma}(x) \stackrel{x \rightarrow \infty}{\longrightarrow}$ $2\left(x F-\varepsilon_{N_{\sigma} \sigma}\right)$, leading to a cancellation of both terms inside the square-root.

As a conclusion of the analysis in two previous paragraphs, let us emphasize that both our $v_{x \sigma}(\mathbf{r})$ and $v_{x \sigma}^{\mathrm{AKK}}(\mathbf{r})$ reproduce the correct asymptotic limit of the exact exchange potential, but in different ways. On the other side, $v_{x \sigma}^{\mathrm{AKK}}(\mathbf{r})$ is system-dependent, being only valid for atomic systems in presence of a bias, while our exchange potential is system-independent, being valid for any 3D system, in the presence of any electric and/or magnetic fields (see below). Also it is better suited for dealing with currents. Moreover, our potential does not require explicit knowledge of external fields (as it should be for any standard density functional) and/or KS eigenenergies - only (occupied) KS orbitals are needed.

In Fig. 2 we show the exchange potentials for a fully spin-polarized four-electron "harmonium", i.e., a 3D harmonic oscillator with a radial external potential $v_{\text {ext }}(r)=\omega^{2} r^{2} / 2$, where $\omega=1$ a.u. This type of potential could be used as a realistic model for quantum dots, i.e., electrons confined in atomic clusters or semiconductor heterostructures. We have also set an external, uniform magnetic field to $B=300$ a.u., so that the occupied KS states have angular momenta $l=0,-1,-2,-3$ and hence there are orbital currents in the system. The BJ potential (thick dashed line) shows erroneous divergent behavior. Similar divergence appearing in two-dimensional harmonic oscillator has been analyzed in detail in Ref. [29]. It was shown that the linear increase in the BJ potential at large $r$ follows directly from the asymptotic limit of the single-particle wave functions, which, in the case of a parabolic confining potential, decays as $\exp \left(-r^{2}\right)$ in contrast with the atomic wave function that decays as $\exp (-r)$. The situation is the same in the 3D case considered here. The LDA result 


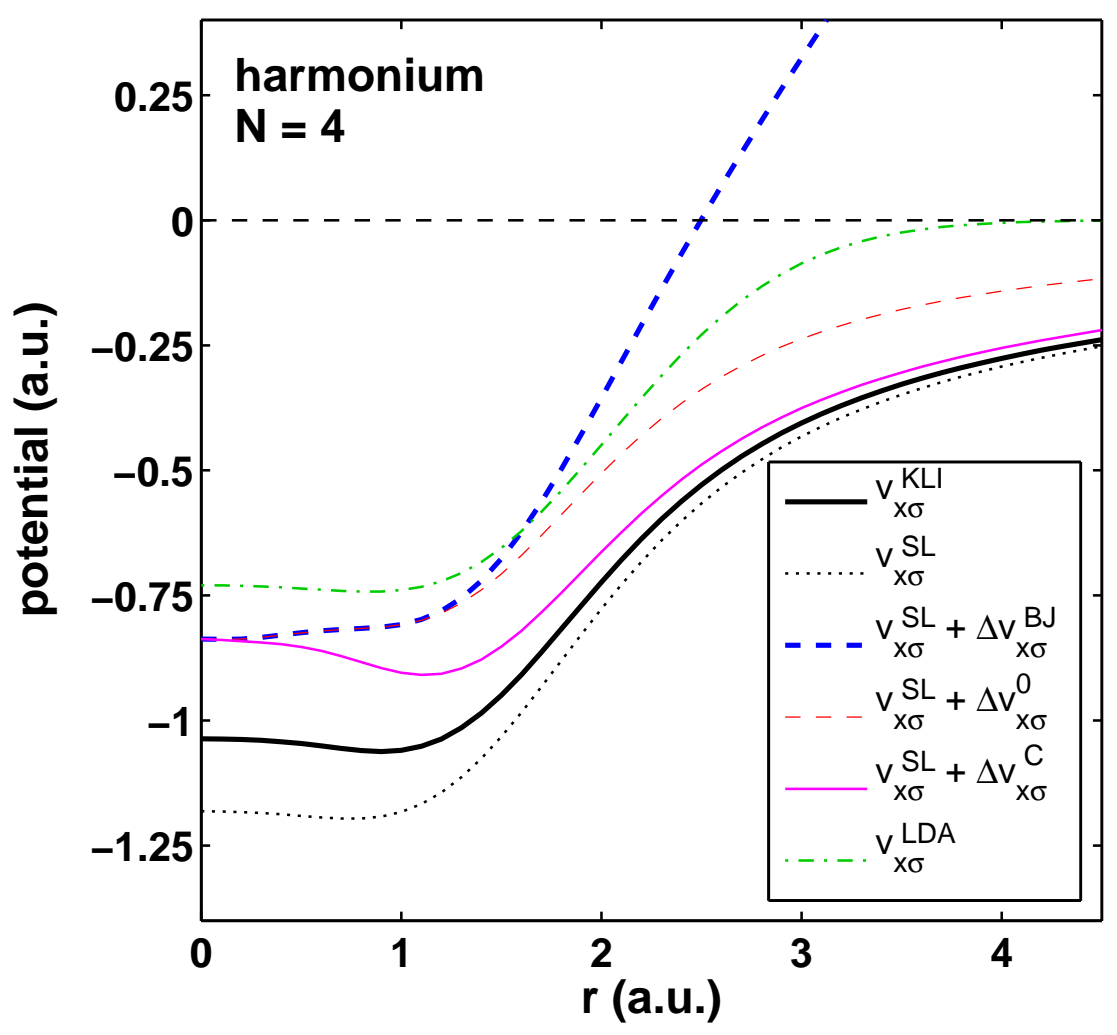

FIG. 2: (Color online) Exchange potentials for a four-electron harmonium in external magnetic field $B=300$ a.u.

(dash-dotted line in Fig. 22), on the other hand, largely underestimates the exchange potential throughout the system. Similar tendency is shown by the BJ potential modified by the gradient term [second term in Eq. (7)] but without the current term [third term in Eq. (7)], i.e., without enforcing the gauge-invariance (thin dashed line marked by $v_{x \sigma}^{\mathrm{SL}}+\Delta v_{x \sigma}^{0}$ ). The closest resemblance of the KLI potential in Fig. 2 is clearly given by the present approximation in Eq. (6). Also it can observed that although the asymptotic limit is very well reproduced, close to the core of this system we still find some deviation, both for the BJ and for our corrected exchange potential. This gives evidence that further improvements may be suggested in future works.

Finally, we verify that the atomic step structure at electronic shells - one of the motivations behind the original Becke-Johnson approximation [19] - is reproduced by the corrected potential. In Fig. 3 we show the exchange potential for a closed-shell neon atom in its ground state with no external fields present. The corrected potential (dashed line) has the step structure at $r \sim 0.3$ a.u. in agreement with the BJ potential (dotted line) and 


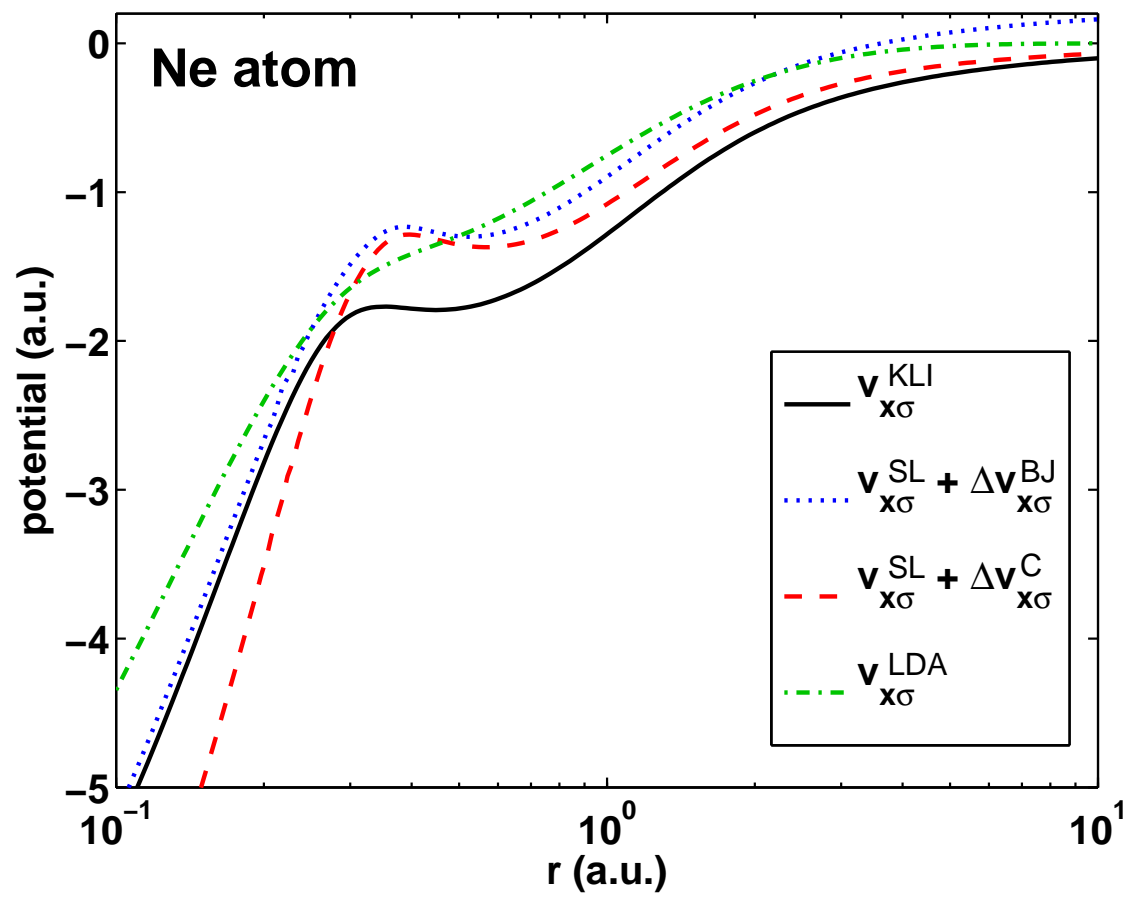

FIG. 3: (Color online) Exchange potential for a closed-shell neon atom in the ground state.

with the KLI potential (solid line). The OEP solution shown in Ref. [19] shows a slightly sharper shoulder than the KLI one, but generally they are very similar, which is in accordance with previous studies in the literature (see, e.g., Ref. [7]). In the exchange-LDA potential (dash-dotted line) the step (or shoulder) structure is missing. Note that for consistency with the previous results we have not imposed a shift to the BJ potential, which is a prerequisite having different definitions [19, 22]. Secondly, we point out that Fig. 3 results from self-consistent calculations for all potentials, respectively. The difference from the non-self-consistent results, obtained by using the KLI orbitals as the input, was found to be negligible.

We point out that for atoms at small $r$ our potential decreases more strongly than the KLI (or BJ) potential (see Fig. 3). In fact, at $r=0$ the correction term $\Delta v_{x}^{\mathrm{C}}$ is significantly smaller than $\Delta v_{x}^{\mathrm{KLI}}$. This difference is not present at $r=0$ in a harmonic confinement discussed above, since in that case it is easy to show that the correction terms in the BJ and our potential have exactly the same value due to the Gaussian form of the orbitals; this feature can also be observed directly from Fig. 2. Nevertheless, the deviation found in the atomic case close to the nuclei suggests that an additional effort beyond the present contribution may be required. 
Concluding, we have seen that Becke-Johnson potential may dramatically fail when considering systems in external electric and magnetic fields. We have proposed a universal correction which is gauge-invariant for complex Kohn-Sham orbitals and exact for any oneparticle system. The improved approximation is suited for dealing with with current-carrying states, and it also recovers the correct asymptotic behavior of the exact exchange potential for any many-electron system. We have demonstrated the very good performance of the resulting exchange potential by considering a hydrogen chain in an external electric field as well as a four-electron harmonic oscillator in a magnetic field.

\section{Acknowledgments}

We would like to thank Giovanni Vignale for useful discussions. This work was supported by the Academy of Finland, Deutsche Forschungsgemeinschaft, and the EU's Sixth Framework Programme through the ETSF e-I3. C.R.P. was supported by the European Community through a Marie Curie IIF (Grant No. MIF1-CT-2006-040222). S.P. acknowledges support by DOE grant DE-FG02-05ER46203.

\section{Appendix}

We may write the term beyond the Slater contribution to the exchange potential as

$$
\Delta v_{x \sigma}^{\mathrm{C}}(\mathbf{r})=C_{\Delta v}\left[\frac{D_{\sigma}(\mathbf{r})}{\rho_{\sigma}(\mathbf{r})}\right]^{\alpha},
$$

and determine $\alpha$ under the constraint of exact linear scaling [36]. Under uniform scaling of the coordinates, $\mathbf{r} \rightarrow \lambda \mathbf{r}$, the norm-preserving many-body wavefunction is given by $\Psi_{\lambda}\left(\mathbf{r}_{1}, \ldots \mathbf{r}_{N}\right)=\lambda^{3 N / 2} \Psi\left(\lambda \mathbf{r}_{1}, \ldots, \lambda \mathbf{r}_{N}\right)$ (with $\left.0<\lambda<\infty\right)$. As a consequence, the 3D density scales with $\lambda$ as follows: $\rho_{\sigma}(\mathbf{r}) \rightarrow \lambda^{3} \rho_{\sigma}(\lambda \mathbf{r})$. This leads to the result that the $\mathrm{KS}$ orbitals in $3 \mathrm{D}$ are seen to scale as $\varphi_{j \sigma}(\mathbf{r}) \rightarrow \lambda^{3 / 2} \varphi_{j \sigma}(\lambda \mathbf{r})$. Thus, $\tau_{\sigma}(\mathbf{r}) \rightarrow \lambda^{5} \tau_{\sigma}(\lambda \mathbf{r})$, $\nabla \rho_{\sigma}(\mathbf{r}) \rightarrow \lambda^{4} \nabla_{\lambda \mathbf{r}} \rho_{\sigma}(\lambda \mathbf{r})$, and $\mathbf{j}_{p \sigma}(\mathbf{r}) \rightarrow \lambda^{4} \mathbf{j}_{p \sigma}(\lambda \mathbf{r})$. Substituting these relations into Eq. (A.1) yields $\Delta v_{x \sigma}^{\mathrm{C} \lambda}(\mathbf{r})=\lambda^{2 \alpha} \Delta v_{x \sigma}^{\mathrm{C}}(\lambda \mathbf{r})$, which fulfills the linear scaling constraint only if $\alpha=1 / 2$ in agreement with the expression in Eq. (6) .

[1] R. M. Dreizler and E. K. U. Gross, Density Functional Theory (Springer, Berlin, 1990). 
[2] U. von Barth, Phys. Scr. T109, 9 (2004).

[3] J. P. Perdew and S. Kurth, in A Primer in Density Functional Theory, Lecture Notes in Physics Vol. 620, edited by C. Fiolhais, F. Nogueira, and M. Marques (Springer, Berlin, 2003).

[4] R. Sharp and G. Horton, Phys. Rev. 90, 317 (1953).

[5] J. D. Talman and W. F. Shadwick, Phys. Rev. A 14, 36 (1976).

[6] S. Kümmel, L. Kronik, Rev. Mod. Phys. 80, 3 (2008).

[7] T. Grabo, T. Kreibich, S. Kurth, and E. K. U. Gross, in Strong Correlations in Electronic Structure Calculations: Beyond the Local Density Approximations, edited by V. Anisimov (Gordon and Breach, Amsterdam, 2000), p. 203.

[8] S. Ivanov, S. Hirata, and R. J. Bartlett, Phys. Rev. Lett. 83, 5455 (1999).

[9] A. Görling, Phys. Rev. Lett. 83, 5459 (1999).

[10] M. Städele, J. A. Majewski, P. Vogl, and A. Görling, Phys. Rev. Lett. 79, 2089 (1997).

[11] C. Horowitz, S. Rigamonti, and C. R. Proetto, Phys. Rev. Lett. 97, 026802 (2006).

[12] S. Rigamonti and C. R. Proetto, Phys. Rev. Lett. 98, 066806 (2007).

[13] S. Sharma, J. K. Dewhurst, C. Ambrosch-Draxl, S. Kurth, N. Helbig, S. Pittalis, S. Shallcross, L. Nordström, and E. K. U. Gross, Phys. Rev. Lett. 98, 196405 (2007).

[14] S. Sharma, S. Pittalis, S. Kurth, S. Shallcross, J. K. Dewhurst, and E. K. U. Gross, Phys. Rev. B 76, 100401(R) (2007).

[15] D. Ködderitzsch, H. Ebert, and E. Engel, Phys. Rev. B 77, 045101 (2008).

[16] E. Engel, D. Ködderitzsch, and H. Ebert, Phys. Rev. B 78, 235123 (2008).

[17] S. Kümmel and J. P. Perdew, Phys. Rev. Lett. 90, 043004 (2003).

[18] S. Kümmel and J. P. Perdew, Phys. Rev. B 68, 035103 (2003).

[19] A. D. Becke and E. R. Johnson, J. Chem. Phys. 124, 221101 (2006).

[20] A. D. Becke and M. R. Roussel, Phys. Rev. A 39, 3761 (1989).

[21] J. B. Krieger, Y. Li, and G. J. Iafrate, Phys. Rev. A 45, 101 (1992).

[22] R. Armiento, S. Kümmel, and T. Körzdörfer, Phys. Rev. B 77, 165106 (2008).

[23] V. N. Staroverov, J. Chem. Phys. 129, 134103 (2008).

[24] M. Kodera, K. Higuchi, A. Narita, and M. Higuchi, Phys. Rev. A 78, 012501 (2008).

[25] A. P. Gaiduk and V. N. Staroverov, J. Chem. Phys. 128, 204101 (2008).

[26] F. Tran, P. Blaha, and K. Schwarz, J. Phys.: Condens. Matter 19, 196208 (2007). 
[27] N. Umezawa, Phys. Rev. A 74, 032505 (2006).

[28] F. Tran and P. Blaha, Phys. Rev. Lett. 102, 226401 (2009).

[29] S. Pittalis, E. Räsänen, and C. R. Proetto, arXiv:0909.0364 (2009).

[30] Phys. Rev. Lett. 59, 2360 - 2363 (1987)

[31] Phys. Rev. B £37, 10685 - 10696 (1988)

[32] J. Tao, Phys. Rev. B 71, 205107 (2005)

[33] J. Tao and J. P. Perdew, Phys. Rev. Lett. 95, 196403 (2005).

[34] S. Pittalis, E. Räsänen, and E. K. U. Gross, Phys. Rev. A 80, 032515 (2009).

[35] To simplify the analysis we assume that the highest occupied KS orbitals are non-degenerate.

[36] M. Levy and J. P. Perdew, Phys. Rev. A 32, 2010 (1985).

[37] A. D. Becke and K. E. Edgecombe, J. Chem. Phys. 92, 5397 (1990).

[38] T. Burnus, M. A. L. Marques, and E. K. U. Gross, Phys. Rev. A 71, 010501 (R) (2005).

[39] E. Räsänen, A. Castro, and E. K. U. Gross, Phys. Rev. B 77, 115108 (2008).

[40] J. F. Dobson, J. Chem. Phys. 98, 8870 (1993).

[41] S. Pittalis, E. Räsänen, N. Helbig, and E. K. U. Gross, Phys. Rev. B 76, 235314 (2007).

[42] A. D. Becke, Can. J. Chem. 74, 995 (1996).

[43] S. Pittalis, E. Räsänen, C. R. Proetto, and E. K. U. Gross, Phys. Rev. B 79, 085316 (2009).

[44] A. D. Becke, J. Chem. Phys, 88, 1053 (1988).

[45] M. A. L. Marques, A. Castro, G. F. Bertsch, A. Rubio, Comput. Phys. Commun. 151, 60 (2003); A. Castro, H. Appel, M. Oliveira, C. A. Rozzi, X. Andrade, F. Lorenzen, M. A. L. Marques, E. K. U. Gross, and A. Rubio, Phys. Stat. Sol. (b) 243, 2465 (2006).

[46] Note that our definition of $\tau_{\sigma}(\mathbf{r})$ includes an extra factor of two.

[47] The decay of the KS orbitals in the direction of the external electric field is given by the asymptotic decay of the Airy functions. 